\title{
Neurofibromatosis-related phaeochromocytoma: two cases with large tumours and elevated plasma methoxytyramine
}

\author{
Stephanie Teasdale and Elham Reda' \\ Endocrinology, Mater Hospital, South Brisbane, Queensland, Australia and ${ }^{1}$ Endocrinology, Gold Coast University \\ Hospital, Southport, Queensland, Australia
}

Correspondence should be addressed to $\mathrm{S}$ Teasdale

Email

stephanie_teasdale@

hotmail.com

\section{Summary}

We present two cases of adrenal phaeochromocytoma in patients with a previous diagnosis of neurofibromatosis type 1 (NF1). One had an adrenergic phenotype. The other had a more noradrenergic phenotype. Both had large primary tumours, which increases the likelihood of malignancy. Both also had elevated plasma-free methoxytyramine, which has been linked with malignancy even in non-SDHB phaeochromocytomas.

\section{Learning points:}

- Phaeochromocytoma can have varied clinical presentations.

- Methoxytyramine can be useful in the biochemical work-up of both SDHB-positive and SDHB-negative phaeochromocytoma.

- The utility of methoxytyramine as a marker of malignancy in NF1-related phaeochromocytoma is unclear, and cases with elevated titres warrant longer follow-up.

\section{Background}

These cases illustrate the need for clarification of biomarkers for malignancy with different genetic subtypes of phaeochromocytoma.

\section{Case presentation}

\section{Case 1}

A 53-year-old male presented to our emergency department with 3 days of new onset right upper quadrant discomfort. He reported intermittent palpitations with dizziness over several years, sweating for 3 months and $5 \mathrm{~kg}$ weight loss over 3 months. Past medical history included neurofibromatosis type 1 (NF1) diagnosed in childhood, atrial fibrillation diagnosed 3 years earlier that was rate-controlled, and a coronary angiogram a year earlier showing no coronary artery disease. He was a 35-pack-a-year smoker. His mother had also had NF1 and had had large lesions removed from her neck and arms. She had died from breast cancer. He lived alone, had left school at 15 years of age and worked as a cleaner. Examination demonstrated tachycardia 180 b.p.m., normotension 120/80 mmHg. He had widespread cutaneous neurofibromas and café au lait spots. He was jaundiced with a palpable mass in his right upper quadrant. Electrocardiogram (ECG) demonstrated atrial fibrillation. Medications included metoprolol $100 \mathrm{mg}$ b.d., digoxin $250 \mu \mathrm{g}$ daily, diltiazem $180 \mathrm{mg}$ daily and rivaroxaban $20 \mathrm{mg}$ daily. He had not taken any rivaroxaban for 1-2 days leading to his presentation. 


\section{Case 2}

A 29-year-old female presented with severe stabbing headache and palpitations, followed by severe central chest pain, shortness of breath and diaphoresis. This was on the background of 5 years intermittent headaches preceded by palpitations of a few minutes' duration. Her blood pressure when asymptomatic had once been 200/120 mmHg several months prior to her presentation, but this wasn't further investigated. She had also had a 2-year history of mild right flank discomfort. She had a background of NF1 diagnosed on the basis of a family history (mother, two brothers and niece) and café au lait spots noted in her early 1920s. Her two brothers had marked cutaneous lesions, and her niece had a tumour in her eye. She took no medications.

She was slightly hypertensive at $150 / 100 \mathrm{mmHg}$, with tachycardia 115 b.p.m. There were four café au lait spots and no neurofibromas. Remaining examination was unremarkable. ECG demonstrated a sinus tachycardia, with ST and T wave changes concerning for ischaemia (ST depression of $1 \mathrm{~mm}$ in inferior leads, ST elevation $1 \mathrm{~mm}$ in V3 initially, then deep $\mathrm{Q}$ waves and $\mathrm{T}$ wave inversion in $\mathrm{V} 1, \mathrm{~V} 2$, I and aVL). Troponin I was $3.1 \mu \mathrm{g} / \mathrm{l}$ on presentation, dropping to $1.9 \mu \mathrm{g} / 110 \mathrm{~h}$ later $(<0.02)$. Chest pain resolved with morphine.

\section{Investigation}

\section{Case 1}

Investigations demonstrated normal serum electrolytes, reduced renal function estimated glomerular filtration rate (eGFR) 68, elevated bilirubin $91 \mu \mathrm{mol} / 1$ and mildly deranged liver enzymes with (ALP 93, GGT 153, ALT 60, AST 57 and LD $485 \mathrm{U} / \mathrm{l})$. Lipase was not elevated. Abdominal ultrasound demonstrated a $6 \times 5 \mathrm{~cm}$ solid cystic lesion that may have been renal or adrenal. Abdominal computed tomography (CT) demonstrated an $8 \times 5.6-\mathrm{cm}$ heterogeneous cystic right adrenal mass that was likely a phaeochromocytoma, plus a $1.5-\mathrm{cm}$ duodenal-jejunal flexure lesion possibly a carcinoid, with intussusception, a $3 \times 4 \mathrm{~cm}$ perirectal lesion likely leiomyoma, small splenic infarcts (possibly secondary to emboli from nonanticoagulated atrial fibrillation), plus multiple neurocutaneous nodules, bullous lung disease and interstitial fibrosis which were consistent with NF1. Subsequent plasma biogenic amines demonstrated marked elevation in free normetadrenaline $12000 \mathrm{pmol} / \mathrm{l}$ (<900), free metadrenaline $7650 \mathrm{pmol} / 1 \quad(<500)$ and free 3-methoxytyramine $565 \mathrm{pmol} / \mathrm{l}(<110)$. Urinary 5-hydroxyinoleacetic acid was not elevated. Cortisol was not elevated. Thyroid function was normal. Iodine-123-metaiodobenzylguanidine $\left({ }^{123}\right.$ I-MIBG) scan showed uptake in the adrenal lesion, and not at any other site. A transthoracic echo showed atrial fibrillation, but normal heart structure.

\section{Case 2}

Abdominal CT demonstrated a $7.7 \times 5.4 \times 5.7-\mathrm{cm}$ lesion most likely to have originated from the right adrenal gland, demonstrating heterogeneous arterial enhancement. The differential included phaeochromocytoma, adrenal carcinoma, or atypical adrenal adenoma. There were also three liver lesions, one $2.3 \times 1.9 \times 2.8-\mathrm{cm}$ arterially enhancing, multilobulated lesion in segment 2 and two subcentimeter lesions arterially hyperenhancing in the right lobe. The liver lesions were of indeterminate aetiology, and differential included metastatic disease, focal nodular hyperplasia or hepatic adenoma. She proceeded to plasma biogenic amines, which showed free normetadrenaline $75000 \mathrm{pmol} / 1$ $(<900)$, free metadrenaline $5970 \mathrm{pmol} / \mathrm{l}(<500)$ and free 3 -methoxytyramine $436 \mathrm{pmol} / \mathrm{l}(<110)$. Plasma dopamine was also elevated $305 \mathrm{pmol} / 1 \quad(<250)$. Magnetic resonance imaging (MRI) demonstrated the adrenal lesion to be compatible with phaeochromocytoma. The liver segment 2 and segment 6 lesions were barely detectable on MRI, and the aetiology remained wide. ${ }^{123}$ I-MIBG showed uptake in the adrenal lesion only. A transthoracic echocardiogram showed normal left ventricular (LV) size, left ventricular ejection fraction (LVEF) $45-50 \%$ and apical and mid-segmental hypokinesis with spared basal function, consistent with Takotsubo cardiomyopathy.

Given the known diagnosis of neurofibromatosis in each case, specialist genetics advice was not to perform further genetic analysis, as the yield would be very low. It was assumed both cases were SDHB-negative.

\section{Treatment}

\section{Case 1}

He had intermittent tachycardia despite commencing diltiazem $180 \mathrm{mg}$ daily and recommencing digoxin $250 \mu \mathrm{g}$ daily. Heart rate ranged 70-180 b.p.m. with blood pressure 90-140/70-80 mmHg. A single dose of prazosin $0.5 \mathrm{mg}$ was given, which was very effective at controlling his tachycardia, but this was ceased after subsequent bradycardia 50-60 b.p.m. and resting hypotension 90/70 mmHg. Labetalol $100 \mathrm{mg}$ b.d. was commenced, 
but heart rate remained elevated at around 130 b.p.m. When the diagnosis of phaeochromocytoma was made, the more traditionally used phenoxybenzamine $10 \mathrm{mg}$ b.d. was commenced. There was ongoing tachycardia and intermittent atrial fibrillation despite up-titration of diltiazem to 240 then $360 \mathrm{mg}$ daily. He was never hypertensive, but rather had episodes of hypotension in attempts to control his heart rate. He was able to tolerate up-titration to $30 \mathrm{mg}$ t.d.s. phenoxybenzamine and metoprolol $100 \mathrm{mg}$ b.d., achieving target blood pressure and heart rate. Surgery was uncomplicated.

\section{Case 2}

She was commenced on phenoxybenzamine and verapamil and at day 9 was switched to metoprolol. She was able to tolerate up-titration to $80 / 40 / 80 \mathrm{mg}$ phenoxybenzamine over 2 months. The high dose of alpha blockade was requested by the operating surgeon. This did require a high dose of metoprolol, 100/50/100 mg, to control the ensuing tachycardia. Surgery was uncomplicated.

\section{Outcome and follow-up}

\section{Case 1}

Histopathology showed elevated Ki67 >3\%, phaeochromocytoma of the adrenal gland scaled score (PASS) score of 4 (borderline) and grading system for adrenal phaeochromocytoma and paraganglioma (GAPP) score of 4 (moderately differentiated). He continued to present to hospital with episodes of rapid atrial fibrillation, and 2 months post surgery remained on $75 \mathrm{mg}$ metoprolol, plus digoxin and rivaroxaban.

\section{Case 2}

Histopathological scoring was reassuring with Ki67 2\%, PASS score 0 and GAPP score 2 (well-differentiated). Postoperative catecholamines and metanephrines had normalised. One month post surgery she still required $100 \mathrm{mg}$ b.d. metoprolol to control her tachycardia, possibly as a result of pre-operative phenoxybenzamine.

\section{Discussion}

\section{Adrenergic phenotype}

Our cases were typical of NF1-related phaeochromocytomas in their elevated levels of adrenaline and adrenaline metabolites. Our two cases differed from each other in the degree of elevation of noradrenaline and noradrenaline metabolites (case 1 , normetadrenaline $\times 13$ ULN and metadrenaline $\times 15$ ULN vs case 2 , normetadrenaline $\times 80$ ULN and metadrenaline $\times 11$ ULN). This is most likely the reason for their different phenotypes.

Case 1 had refractory tachycardia and no hypertension, a phenotype that has been described for adrenalinesecreting phaeochromocytomas, and thought to be at least partly due to excess activity on $\beta 2$ receptors causing vasodilatation (1). This theory is supported by a case of adrenaline-secreting phaeochromocytoma with recurrent syncope from postural hypotension, which resolved with propranolol. The tachycardia and hypotension in case 1 was ultimately alleviated with non-selective alpha and cardioselective beta blockade, but it is interesting to conjecture whether heart rate may have been controlled more rapidly had the patient been continued on alpha-1 selective alpha blockade with Prazosin, and thus avoided the several days of difficult-to-control tachycardia that comes from removing the negative regulation of noradrenaline secretion with alpha-2 blockade.

Case 2, on the other hand, presented more classically, and had less tachycardia and a degree of hypertension. Case 2 also had Takotsubo cardiomyopathy. NF1 can predispose to structural cardiac abnormalities not just via excess catecholamines in the context of phaeochromocytoma, but also by deposition of neurofibromin leading to left ventricular hypertrophy (2).

\section{Diagnosing malignancy and malignant potential}

Our two cases demonstrated clear ${ }^{123}$ I-MIBG uptake in the adrenal primaries, but no convincing uptake elsewhere. It was decided in each case to proceed to resection of the primary first before considering alternative imaging modalities such as ${ }^{18} \mathrm{~F}$-FDOPA PET (that might be considered for NF1 tumours suspicious for malignancy per the EANM 2012 guidelines) (3). Unfortunately, normalisation of catecholamine secretion post-operatively does not rule out malignancy.

Historically there have been no histological or immunohistochemical criteria that could distinguish malignant from benign disease. Ki67 index is specific but not sensitive. A recent publication suggested that a combination of Ki67 and PASS has utility, and tumours with Ki67 $>2 \%$ and PASS $>6$ should be followed closely (4). The GAPP score is a promising new system for assigning risk of malignancy to phaeochromocytomas according to histopathological and other criteria (5). By Ki67 and GAPP scores, case 1 should be watched 
closely, but case 2 could be deemed very low-risk for malignancy.

Plasma-free methoxytyramine has been demonstrated to be a discriminating biomarker for metastatic PPGLs, additive to tumour size and extra-adrenal tumour location, and irrespective of SDHB status (6). There have been no studies that look at the utility of the titre of plasma-free methoxytyramine in non-SDHB genetic subsets. Plasma-free methoxytyramine was not significantly greater in NF1 tumours than in tumours from other hereditary syndromes, and was trending to be lower than from tumours without an established mutation or hereditary syndrome (7). This suggests that the elevated levels of plasma methoxytyramine seen in our two cases is atypical for specifically NF1-related phaeochromocytomas. It is not clear whether this is predictive of malignancy.

The mechanism for elevation of methoxytyramine in SDHB-negative tumours is unclear, and may be distinct from that for SDHB-positive phaeochromocytomas (7) (8).

\section{Declaration of interest}

The authors declare that there is no conflict of interest that could be perceived as prejudicing the impartiality of the research reported.

\section{Funding}

This research did not receive any specific grant from any funding agency in the public, commercial or not-for-profit sector.

\section{Patient consent}

Written informed consent has been obtained from the patients for publication of the submitted article.

\section{Author contribution statement}

Dr S Teasdale was involved in the clinical care of the patients and authored the case descriptions and the discussion. Dr E Reda was the endocrinologist who was responsible for the clinical care of the patients.

\section{References}

1 Pacak K 2011 Pheochromocytoma: a catecholamine and oxidative stress disorder. Endocrine Regulations 45 65-90. (doi:10.4149/ endo_2011_02_65)

2 Zinnamosca L, Petramala L, Cotesta D, Marinelli C, Schina M, Cianci R, Giustini S, Sciomer S, Anastasi E, Calvieri S et al. 2011 Neurofibromatosis type 1 (NF1) and pheochromocytoma: prevalence, clinical and cardiovascular aspects. Archives of Dermatological Research 303 317-325. (doi:10.1007/s00403-010-1090-z)

3 Taïeb D, Timmers HJ, Hindié E, Guillet BA, Neumann HP, Walz MK, Opocher G, de Herder WW, Boedeker CC, Krijger RR et al. 2012 EANM 2012 guidelines for radionuclide imaging of phaeochromocytoma and paraganglioma. European Journal of Nuclear Medicine and Molecular Imaging 39 1977-1995. (doi:10.1007/s00259-012-2215-8)

4 Białas M, Okoń K, Dyduch G, Ciesielska-Milian K, Buziak M, Hubalewska-Dydejczyk A \& Sobrinho-Simoes M 2013 Neuroendocrine markers and sustentacular cell count in benign and malignant pheochromocytomas - a comparative study. Polish Journal of Pathology 2 129-135. (doi:10.5114/pjp.2013.36004)

5 Kimura N, Takayanagi R, Takizawa N, Itagaki E, Katabami T, Kakoi N, Rakugi H, Ikeda Y, Tanabe A, Nigawara T et al. 2014 Pathological grading for predicting metastasis in phaeochromocytoma and paraganglioma. Endocrine-Related Cancer 21 405-414. (doi:10.1530/ERC-13-0530)

6 Eisenhofer G, Lenders JW, Siegert G, Bornstein SR, Friberg P, Milosevic D, Mannelli M, Linehand WN, Adams K, Timmers HJ et al. 2012 Plasma methoxytyramine: a novel biomarker of metastatic pheochromocytoma and paraganglioma in relation to established risk factors of tumour size, location and SDHB mutation status. European Journal of Cancer $\mathbf{4 8}$ 1739-1749. (doi:10.1016/j.ejca.2011.07.016)

7 Eisenhofer G, Pacak K, Huynh TT, Qin N, Bratslavsky G, Linehan WM, Mannelli M, Friberg P, Grebe SK, Timmers HJ et al. 2011 Catecholamine metabolomics and secretory phenotypes in phaeochromocytoma. Endocrine-Related Cancer 18 97-111. (doi:10.1677/ERC-10-0211)

8 Van Berkel A, Lenders JW \& Timmers HJ 2014 Diagnosis of endocrine disease: biochemical diagnosis of phaeochromocytoma and paraganglioma. European Journal of Endocrinology 170 R109-R119. (doi:10.1530/ EJE-13-0882)

Received in final form 18 June 2015

Accepted 15 July 2015 